\title{
Prevalence of active trachoma and associated factors among children aged 1 to 9 years in rural communities of Lemo district, southern Ethiopia: community based cross sectional study
}

\author{
Endale WoldeKidan ${ }^{1}$, Deresse Daka ${ }^{2}$, Deresse Legesse ${ }^{3}$, Tariku Laelago ${ }^{4 *}$ (D) and Bealu Betebo ${ }^{1}$
}

\begin{abstract}
Background: Trachoma, caused by Chlamydia trachomatis is the leading infectious cause of blindness. It is transmitted via personal contact with infected ocular and nasal secretions by hands, fomites and eye- seeking flies. Active trachoma is more common among children aged 1 to 9 years. The objective of this study was determining the prevalence of active trachoma and associated factors among children aged 1 to 9 years in rural community of Lemo district.

Methods: Community-based cross-sectional study was conducted from March to April, 2018 in rural community of Lemo district. Multistage sampling technique was used to select 589 study participants. Data were collected by using structured pre-tested questionnaire, physical examination and observation. Binocular loupe was used to identify active trachoma cases. The data were entered by using EPi-data version 3.1 and analyzed by SPSS. Binary logistic regression was used to assess factors associated with active trachoma. Variables with $p$-value $<0.05$ in the multivariable analysis were used to declare significance of association.

Result: Eighty seven (15.2\%) children were positive for active trachoma. Absence of solid waste disposal pit (AOR= 2.20, 95\% Cl (1.12-4.37), do not use latrine as reported by respondent ( $\mathrm{AOR}=7.53,95 \% \mathrm{Cl}$ (2.86-19.84), do not use soap for face washing as reported by respondent ( $A O R=2.3,95 \% \mathrm{Cl}(1.32-4.12)$, washing face frequency as reported by respondent $(A O R=1.86,95 \% \mathrm{Cl}(1.06-3.26)$, and family size greater than five ( $A O R=1.96,95 \% \mathrm{Cl}(1.06-3.67)$ were significantly associated with active trachoma.

Conclusion: Active trachoma among children aged 1 to 9 years is high. Do not use latrine, do not use soap for face washing, and face washing frequency in a day as reported by respondents and family size were associated with active trachoma. Access to adequate water and sanitation can be important components in working towards eliminating trachoma as a public health problem. Therefore, prompt measures must be taken by concerned bodies to increase access to adequate water and sanitation facilities.
\end{abstract}

Keywords: Active trachoma, Prevalence, Children, Factors

\footnotetext{
* Correspondence: tarikulalago@gmail.com

${ }^{4}$ Department of nursing, Wachemo University, Durame campus, Durame,

Ethiopia

Full list of author information is available at the end of the article
}

(c) The Author(s). 2019 Open Access This article is distributed under the terms of the Creative Commons Attribution 4.0 International License (http://creativecommons.org/licenses/by/4.0/), which permits unrestricted use, distribution, and reproduction in any medium, provided you give appropriate credit to the original author(s) and the source, provide a link to the Creative Commons license, and indicate if changes were made. The Creative Commons Public Domain Dedication waiver (http://creativecommons.org/publicdomain/zero/1.0/) applies to the data made available in this article, unless otherwise stated. 


\section{Background}

Trachoma, caused by particular serovars of Chlamydia trachomatis (C. trachomatis), is the leading infectious cause of blindness [1]. It is transmitted in ocular and nasal secretions that are passed from person to person on fingers, fomites (such as bedding and washcloths) and eye-seeking flies (particularly Musca sorbens) [2]. C. trachomatis infection is associated with an inflammatory conjunctivitis known as "active trachoma". Repeated episodes of active trachoma can result in eyelid scarring, which in some individuals leads to trachomatous trichiasis, in which one or more eyelashes are diverted to touch the eye [2].

C. trachomatis infections of the eye are commonest in young children. Children are the main reservoirs of infection. Active trachoma cases are more common among children aged 1 to 9 years [3]. Children are frequently infected with $C$. trachomatis due to their tendency to have close contact with others [4].

The diagnosis of active trachoma is a clinical diagnosis based on the WHO (world health organization) simplified grading system. Trachomatous inflammation - follicular (TF) and Trachomatous inflammation- Intense (TI) are indications of active trachoma and are usually found in children, but may occasionally occur in older persons $[3,5]$. Each sign is individually graded as being absent or present. One or more signs can, and often do, occur together $[3,5]$.

Globally, an estimated 36 million people were blind, 216.6 million people had moderate to severe visual impairment and 188.5 million had mild visual impairment. The estimated number of blind people increased by 17.6\%, from 30.6 million in 1990 to 36.0 million in 2015 . The number of people with moderate and severe visual impairment also increased, from 159.9 million in 1990 to 216.6 million in 2015 [6]. Trachoma is responsible for blindness or visual impairments of about 1.9 million people [6].

About 165.1 million people existed in areas in which the TF occurrence in children aged 1-9 years was greater than or equal to $5 \%$ at some time during 2017. These people were fit for operation of the A, F and $\mathrm{E}$ components of the SAFE (Surgery, Antibiotic, Facial cleanliness and Environmental improvement) strategy for trachoma elimination. From 165.1 million people, 89\% (146.3 million) were in WHO's African region. Ethiopia accounted 69.8 million cases of WHO's African regions. By 17 April 2018,157.7 million people were living in districts in which the TF prevalence was greater than or equal to $5 \%$ [7].

Different study findings in Ethiopia showed that the prevalence of active trachoma among children aged 19 years is high. The prevalence of active trachoma among children aged 1-9 was 29\% in Amhara regional state [8], 12\% in Dangla town [9], 25.2\% in Kersa district, and $12.1 \%$ in Gondar zuria district of Ethiopia [10]. A region-wide study conducted in Southern nations, nationalities and peoples' region showed the prevalence of active trachoma among children 1-9 years old 25.9\%. The highest prevalence, 48.5\% were observed in Amaro and Burji districts of Segen zone followed by Hadiya zone, which was $45 \%$ [11]. Study done in Zala district of Ethiopia showed that $36.5 \%$ of children aged 1 to 9 years had active trachoma [12]. Furthermore, the study carried out in Loma district of Ethiopia indicated that $75.4 \%$ of children had active trachoma [13].

The presence of active trachoma is associated with different factors. Income, educational status of parents, family size of households $(\mathrm{HH})$, and age of children were factors associated wth active trachoma [4, 10, 14-17]. Washing face $[8,16]$, unclean face of child $[12,13]$, use soap for face washing $[9,16,18]$, lack of access to pipe water [19], walking distance to fetch water [20], and lack of latrine $[21,22]$ were also associated with active trachoma. Moreover, disposing solid wastes and liquid on open field, using animal dung for cooking, and absence of separate room for animals were associated with presence of active trachoma [13, 18, 20, 23, 24].

The high burden of trachoma in Ethiopia in general and in Hadiya zone in particular calls for collecting a further district-specific data and comprehensive efforts for designing and expanding intervention programs. Prevention of trachoma related blindness requires a number of interventions. The WHO and their partners endorse SAFE strategy for trachoma control [4, 25]. Despite the implementation of SAFE strategy in study area, active trachoma still remains to be major public health problem. Moreover, in study area no report has been documented so far on prevalence and associated factors of active trachoma among children aged of 1-9 years. Therefore, this study determined the prevalence of active trachoma and associated factors among children aged 1 to 9 years in the rural community of Lemo district.

\section{Methods \\ Study setting}

The study was conducted in Lemo district, Hadiya Zone, Southern Ethiopia. The district is $230 \mathrm{~km}$ from Addis Ababa in the southwest direction. The district has two agro-climatic divisions: 10\% kola (low land) and 90\% woynedega (mid land). The projected total population of the district is 153,469 in 2018 . Of this total population, children aged 1-9 years are 40,117. There are 33 rural and 2 urban Keble in the district. Concerning the health infrastructure, there are 7 government health centers, 33 health posts and 17 private health facilities. 


\section{Study design and period}

A community based cross-sectional study was carried out from March to April, 2018.

\section{Study participants}

The source population of the study was children age 1 to 9 years. The study participants were children aged 1 to 9 years in selected $\mathrm{HH}$. Children who had serious sickness and refused $\mathrm{HH}$ to participate in study were excluded from the study.

\section{Sample size}

The sample size was calculated by using EPi-info software with a single population proportion with assumptions; 95\% confidence interval, 0.05 margin of error, and prevalence of active trachoma, 36.7\% [12], design effect 1.5 and $10 \%$ non-response rate. Based on the above assumption, the last sample size became $589 \mathrm{HH}$.

\section{Sampling procedure}

The study participants were selected by using a multistage sampling technique. In the first stage, 10 Keble were selected from the total 33 rural Keble by simple random sampling method. The reason for selecting only 10 Keble was cost and feasibility issue. The final sample size was proportionally allocated to the selected ten Keble based on their number of $\mathrm{HH}$ with children 1-9 years. Systematic sampling technique was used to select $\mathrm{HH}$ from each selected Keble. To determine the interval of $\mathrm{HH}$ in selected Keble, $\mathrm{k}^{\text {th }}$ value was used. In case when $\mathrm{HH}$ had more than one eligible child, one child was selected by lottery methods.

\section{Data collection processes and measurements}

Data were collected by face-to-face interview, observation, and clinical eye examination by using pretested interviewer-administered structured questionnaire. The questionnaire was developed by reviewing different literatures. The questionnaire was first prepared in English and translated to Hadiyisa, and then translated back to English in order to ensure consistency (Additional file 1). Three integrated eye care worker (IECW) who have been trained on management and diagnosis of eye were assigned to perform eye examination. The IECWs are tropical data certified trachoma graders. Each eye was examined separately by using binocular examination loupes lenses $(\times 2.5)$. The examination of the eye was done by careful inspection of eye lashes, cornea, limbus, eversion of the upper lid and inspection of the tarsal conjunctiva. The reporting of eye examination results was based on the WHO grading system [3].

The dependent variable of the study was presence of active trachoma. It was assessed by TF and TI $[3,5]$. TF is defined as the presence of five or more follicles of greater than $0.5 \mathrm{~mm}$ in diameter in the central part of upper tarsal conjunctiva. TI is pronounced inflammatory thickening of the tarsal conjunctiva that obscures more than half of the normal deep tarsal vessels. Active trachoma was defined as the presence of TF or TI according to the WHO Guidelines.

The independent variables of the study comprised socio demographic and economic factors, $\mathrm{HH}$ factors, environmental and child factors.

Variables assessed by questioning a $\mathrm{HH}$ resident included: sex of $\mathrm{HH}$ head, education status of $\mathrm{HH}$ head, occupation of $\mathrm{HH}$ head, family size and number children aged 1 to 9 years, marital status, religion, and ethnicity. Sex of child, age of child, frequency of face washing and using soap for face washing were also assessed by questioning a $\mathrm{HH}$ resident. Furthermore, time taken to fetch water, amount of water for domestic consumption, ownership of cattle and utilization of latrine were assessed by questioning a $\mathrm{HH}$ resident.

Variables assessed through observation by the research team included: number of rooms, separate room for cattle and cooking, presence of window in the cooking room, availability of latrine, type of latrine and distance of latrine from living house. Solid waste system availability, liquid waste disposal, presence of hand washing facility and feces seen in the compound were also assessed through observation by the research team. Moreover, cleanliness of child face and fingers, ocular discharge and nasal discharge were also assessed through observation by the research team.

\section{Clean face}

Absence of an ocular or nasal discharge on the face of child at the time of the visit.

\section{Clean finger}

Absence of dirty materials on fingers and fingers' nail.

\section{Data quality control}

Before beginning data collection, 2 days training was given for 3 data collectors, 3 trachoma examiners and 2 supervisors. Before the actual data collection, the examination of eye with questionnaire was pre-tested on 5\% of final sample size in an adjacent Keble, which was out of study area. During the course of the data collection, data collectors were intensively supervised at each site. The completeness and accuracy of data was checked at the end of each day.

\section{Statistical analysis}

The data were cleaned to check for its consistency, completeness and the existence of missed values. Then, it was entered into EPi-data and exported to SPSS version 20 for analysis. Descriptive findings were calculated by 
using frequencies, percentages, and summary statistics. Binary logistic regression model was fitted to assess factors associated with active trachoma. Variables with $P$-value $<0.25$ in bivariate analysis were included in multivariable analysis to control confounding factors. Odds ratio at $95 \% \mathrm{CI}$ was computed to show strength of association between dependent and independent variables. Variables with $P$-value $<0.05$ in the multivariable analysis were used to declare significance of association.

\section{Results}

\section{Socio demographic and economic characteristics}

A total of 574 children aged 1 to 9 years were included in this study with response rate of $97.4 \%$.

Majority, 521 (90.8\%) HH heads were males. More than half $\mathrm{HH}$ heads, 311 (54.2\%) attended primary schools. The occupation of $\mathrm{HH}$ head showed, 431 (75.1\%) were farmers. One hundred eight six (32.4\%) $\mathrm{HH}$ had more than two children aged 1-9 years. The median children less or equal to 9 years were 2 with minimum of 1 and maximum of 4 . The family size was greater than five, for $384(66.9 \%) \mathrm{HH}$. The median size of family members was 6 persons, with minimum of 3 and maximum of 12 . Concerning to religion, 357 (62.2\%) respondents were Protestants. Regarding to ethnicity, 558 (97.2\%) were Hadiya. About, 549 (95.6\%) $\mathrm{HH}$ heads were married (Table 1).

\section{$\mathrm{HH}$ factors}

In $360(62.7 \%) \mathrm{HH}$, the family members live in one to two rooms. About, 223(38.9\%) HH used 41-601 water for domestic use. The median amount of water used for domestic use was 601 with minimum of 20 and maximum of $100 \mathrm{l}$. Regarding to time taken on foot travel from water sources, $413(72 \%) \mathrm{HH}$ spent $<=30 \mathrm{~min}$ to collect water. The median distance to travel to get water was $25 \mathrm{~min}$ with minimum of 10 and maximum of $60 \mathrm{~min}$. Four hundred forty two (85.6\%) HH kept domestic animals inside the living houses. Three hundred seventy four (65.2\%) $\mathrm{HH}$ cooked their food inside the living rooms. About, 101(52.3\%) cooking rooms had windows (Table 2).

\section{Environmental health related factors}

Majority of $\mathrm{HH}, 561$ (97.7\%) had latrine. More than half, 318 (56.7\%) latrine types was covered pit latrine. About, 548 (99.5\%) family members utilized latrines. Only, 37 (6.6\%) HH had larine with hand washing facility. Latrine distance from living house was greater than $10 \mathrm{~m}$ for $482(85.9 \%) \mathrm{HH}$. The median distance of latrine from living room was $15 \mathrm{~m}$ with minimum of 7 and maximum of $30 \mathrm{~m}$. Merely, 168(29.3\%) HH had solid waste disposal pit. Liquid waste disposal is near house for $411(71.6 \%) \mathrm{HH}$. In $14(2.4 \%) \mathrm{HH}$, feces were seen in the compound (Table 3 ).
Table 1 Socio demographic and economic characteristics of $\mathrm{HH}$ heads in rural Lemo district, Southern Ethiopia, 2018

\begin{tabular}{|c|c|c|}
\hline Variables & Frequency $(N=574)$ & Percent \\
\hline \multicolumn{3}{|l|}{ Sex of HH head } \\
\hline Male & 521 & 90.8 \\
\hline Female & 53 & 9.2 \\
\hline \multicolumn{3}{|c|}{ Educational status of $\mathrm{HH}$ head } \\
\hline No formal education & 137 & 23.9 \\
\hline Primary education & 311 & 54.2 \\
\hline Secondary education & 114 & 19.9 \\
\hline College and above & 12 & 2.1 \\
\hline \multicolumn{3}{|l|}{ Occupation of $\mathrm{HH}$ head } \\
\hline Farmer & 431 & 75.1 \\
\hline Craftsman & 30 & 5.2 \\
\hline Housewife & 55 & 9.6 \\
\hline Merchant & 52 & 9.1 \\
\hline Others $^{a}$ & 6 & 1 \\
\hline \multicolumn{3}{|l|}{ Family size } \\
\hline$\leq 5$ family members & 190 & 3.1 \\
\hline$>5$ family members & 384 & 66.9 \\
\hline \multicolumn{3}{|c|}{ Number of 1 to 9 years children } \\
\hline 1-2 children & 388 & 67.6 \\
\hline$\geq 3$ children & 186 & 32.4 \\
\hline \multicolumn{3}{|l|}{ Marital status } \\
\hline Married & 549 & 95.6 \\
\hline Widowed & 23 & 4 \\
\hline Divorced & 2 & 0.3 \\
\hline \multicolumn{3}{|l|}{ Religion } \\
\hline Orthodox & 135 & 23.5 \\
\hline Protestant & 357 & 62.2 \\
\hline Muslim & 46 & 8 \\
\hline Catholic & 36 & 6.3 \\
\hline \multicolumn{3}{|l|}{ Ethnicity } \\
\hline Hadiya & 558 & 97.2 \\
\hline Gurage & 10 & 1.7 \\
\hline Kambeta & 4 & 0.7 \\
\hline Silte & 2 & 0.3 \\
\hline
\end{tabular}

Other $^{\mathrm{a}}$ Daily labor.

\section{Child factors}

More than half, 296(51.6\%) children were males. Regarding to age distribution of children, 372(64.8\%) were aged 1-5 years. The mean age of children was 4.74 years. Three hundred fifty four (61.7\%) children washed their face once a day. Children's facial cleanness indicated that $457(79.6 \%)$ children faces were unclean. Fifty one (8.9\%) children had ocular discharge. Sixty (10.5\%) children had nasal discharge. Regarding cleanness of children fingers, 
Table 2 The HH condition in rural communities of Lemo District Ethiopia, 2018

\begin{tabular}{|c|c|c|}
\hline Variables & Frequency & Percent \\
\hline \multicolumn{3}{|l|}{ Rooms in $\mathrm{HH}, \mathrm{N}=574$} \\
\hline $1-2$ rooms & 361 & 62.9 \\
\hline Three and above rooms & 213 & 37.1 \\
\hline \multicolumn{3}{|c|}{$\begin{array}{l}\text { Time taken to fetch water from } \\
\text { the source, } N=574\end{array}$} \\
\hline$\leq 30 \min$ & 413 & 72.0 \\
\hline$>30 \min$ & 161 & 28.0 \\
\hline \multicolumn{3}{|c|}{$\begin{array}{l}\text { Amount of water for domestic } \\
\text { consumption, } N=574\end{array}$} \\
\hline 20-40 Liters & 115 & 20.0 \\
\hline $41-601$ & 223 & 38.9 \\
\hline 61-80 Liters & 159 & 27.7 \\
\hline$>80$ Liters & 77 & 13.4 \\
\hline \multicolumn{3}{|l|}{ Cattle ownership, $N=574$} \\
\hline No & 60 & 10.5 \\
\hline Yes & 514 & 89.5 \\
\hline \multicolumn{3}{|c|}{ Separate room for cattle, $N=514$} \\
\hline No & 440 & 76.7 \\
\hline Yes & 74 & 12.9 \\
\hline \multicolumn{3}{|c|}{ Separate cooking room, $N=574$} \\
\hline No & 374 & 65.2 \\
\hline Yes & 200 & 34.8 \\
\hline \multicolumn{3}{|c|}{ Window for cooking room, $N=200$} \\
\hline No & 99 & 48.7 \\
\hline Yes & 101 & 52.3 \\
\hline
\end{tabular}

only 165 (28.7\%) children fingers were clean. About, 330 (57.5\%) children do not used soap for face washing (Table 4).

\section{Prevalence of active trachoma}

The overall prevalence of active trachoma among children aged 1-9 years was $15.2 \%$. Of total examined children aged 1-9 years, the prevalence of TF and TI was 13.6 and $1.6 \%$, respectively.

\section{Factors associated with active trachoma}

Variables with P-value less than 0.25 in bivariate analysis were transferred to multivariable logic regression analysis. The following variables were included into multivariable logistic regression model by using enter method: utilization of latrine, solid waste disposal pit, frequency of face washing, family size, use soap for face washing, rooms in $\mathrm{HH}$, $\mathrm{HH}$ water consumption, time taken to fetch water age of child, separate cooking room, distance of latrine from living house and number of children age 1 to 9 years. However, in multivariable logistic regression analysis; utilization
Table 3 Environmental health related factors in Lemo district, 2018

\begin{tabular}{|c|c|c|}
\hline Variables & Frequency & Percent \\
\hline \multicolumn{3}{|c|}{ Availability of latrine $N=574$} \\
\hline No & 13 & 2.3 \\
\hline Yes & 561 & 97.7 \\
\hline \multicolumn{3}{|l|}{ Type of latrine $N=561$} \\
\hline Uncovered pit latrine & 243 & 43.3 \\
\hline Covered pit latrine & 318 & 56.7 \\
\hline \multicolumn{3}{|c|}{ Utilization of latrine, $N=561$} \\
\hline No & 22 & 3.9 \\
\hline Yes & 539 & 96.1 \\
\hline \multicolumn{3}{|c|}{$\begin{array}{l}\text { Latrine distance from living } \\
\text { house, } N=561\end{array}$} \\
\hline$\leq 10 \mathrm{~m}$ & 79 & 14.1 \\
\hline$>10 m$ & 482 & 85.9 \\
\hline \multicolumn{3}{|c|}{ Hand washing facility, $N=561$} \\
\hline No & 524 & 93.4 \\
\hline Yes & 37 & 6.6 \\
\hline \multicolumn{3}{|c|}{ Solid waste disposal, $N=574$} \\
\hline No & 406 & 70.7 \\
\hline Yes & 168 & 29.3 \\
\hline \multicolumn{3}{|c|}{ Liquid waste disposal, $N=574$} \\
\hline Near house & 411 & 71.6 \\
\hline Far from house & 163 & 28.4 \\
\hline \multicolumn{3}{|c|}{ Feces in the compound, $N=574$} \\
\hline No & 560 & 97.6 \\
\hline Yes & 14 & 2.4 \\
\hline
\end{tabular}

of latrine as reported by respondent, absence of solid waste disposal pit, face washing as reported by respondents, family size and do not use of soap for face washing as reported by respondents were found independently associated with active trachoma.

$\mathrm{HH}$ that did not have solid waste disposal pit were 2.2 times more likely to be affected by active trachoma than those $\mathrm{HH}$ that had solid waste disposal pit $(\mathrm{AOR}=2.2$, 95\% CI (1.12-4.37).

Families that didn't use latrine were 7.5 times more likely to be affected by active trachoma than those families who utilize latrine (AOR = 7.53, 95\% CI (2.86-19.84).

Children who did not use soap for face washing were 2.3 times were more likely to develop active trachoma than children who used soap for face washing (AOR = 2.3, 95\% CI (1.32 -4.12). Furthermore, children who washed their face once a day were 1.86 times more likely to have active trachoma than those who washed twice and more times $(\mathrm{AOR}=1.86,95 \% \mathrm{CI}$ (1.06-3.26).

children living in $\mathrm{HH}$ with family size greater than five were about 2 times more likely to develop active trachoma than children living children living in $\mathrm{HH}$ 
Table 4 Child features in rural communities of Lemo district Ethiopia, 2018

\begin{tabular}{|c|c|c|}
\hline Variables & Frequency $(N=574)$ & Percent \\
\hline \multicolumn{3}{|l|}{ Sex of child } \\
\hline Male & 296 & 51.6 \\
\hline Female & 278 & 48.4 \\
\hline \multicolumn{3}{|l|}{ Age of child } \\
\hline $1-5$ years & 372 & 64.8 \\
\hline 6-9 years & 202 & 35.2 \\
\hline \multicolumn{3}{|l|}{ Frequency of face washing } \\
\hline Once a day & 361 & 62.9 \\
\hline Twice and more times a day & 213 & 37.1 \\
\hline \multicolumn{3}{|l|}{ Face condition of child } \\
\hline Unclean & 125 & 21.8 \\
\hline Clean & 449 & 78.2 \\
\hline \multicolumn{3}{|l|}{ Ocular discharge } \\
\hline No & 523 & 91.1 \\
\hline Yes & 51 & 8.9 \\
\hline \multicolumn{3}{|l|}{ Nasal discharge } \\
\hline No & 514 & 89.5 \\
\hline Yes & 60 & 10.5 \\
\hline \multicolumn{3}{|l|}{ Cleanness' of the child finger } \\
\hline No & 409 & 71.3 \\
\hline Yes & 165 & 28.7 \\
\hline \multicolumn{3}{|l|}{ Used soap for face working } \\
\hline No & 330 & 57.2 \\
\hline Yes & 244 & 42.5 \\
\hline
\end{tabular}

less or equal to five family size $(\mathrm{AOR}=1.97,95 \% \mathrm{CI}$ (1.06 -3.67) (Table 5).

\section{Discussion}

This study attempted to identify the prevalence and associated factors of active trachoma among children aged 1 to 9 years in rural communities of Lemo district. In current study the prevalence of active trachoma was $15.2 \%$. The study also showed that the prevalence of TF was $13.6 \%$. This greater than the criteria for elimination of trachoma as public health problem, which showed that the prevalence of $\mathrm{TF}<5 \%$ in children aged 1-9 years old [26].

The prevalence of active trachoma in current study is different to the prevalence of studies done in different parts of Ethiopia [8, 9, 11, 12, 14, 17, 21]. The difference could be attributed to difference in study setting, the infrastructure and health care facilities. The other reasons for variations might be due to differences in utilization of sanitary services. The prevalence of active trachoma in current is lower than the study conducted in South nation's nationalities people region, which showed 33.2\% [27]. The lower prevalence of active trachoma in current study may be due to the implementation of SAFE strategy at population level.

The current study highlighted that $\mathrm{HH}$ that did not have solid waste disposal pit were more likely to be affected by active trachoma than those $\mathrm{HH}$ that had solid waste disposal pit. This result is in agreement with study conducted in Gondar zuria district of Ethiopia [23]. Study conducted in Daworo zone of Ethiopia also

Table 5 Factors associated with active trachoma among children aged 1-9 years, rural Lemo district, 2018

\begin{tabular}{|c|c|c|c|c|c|}
\hline \multirow[t]{2}{*}{ Variables } & \multicolumn{2}{|c|}{ active trachoma } & \multirow[t]{2}{*}{ COR (95\% C.I) } & \multirow[t]{2}{*}{ AOR (95\% C.I) } & \multirow[t]{2}{*}{$P$-value } \\
\hline & Yes (n (\%) & No (n (\%) & & & \\
\hline \multicolumn{6}{|c|}{ Utilization of latrine } \\
\hline No & $12(2.1)$ & $10(1.8)$ & $7.78(3.2-18.67)$ & $7.53(2.86-19.84)$ & 0.000 \\
\hline Yes & $72(12.8)$ & $467(83.2)$ & 1 & 1 & \\
\hline \multicolumn{6}{|c|}{ Solid waste disposal pit } \\
\hline No & $74(12.9)$ & $332(57.8)$ & $2.66(1.4-4.9)$ & $2.20(1.12-4.37)$ & 0.024 \\
\hline Yes & $13(2.3)$ & $155(27)$ & 1 & 1 & \\
\hline \multicolumn{6}{|c|}{ Frequency of face washing } \\
\hline Once a day & $63(11)$ & $298(51.9)$ & $1.67(0.363-0.995)$ & $1.86(1.06-3.26)$ & 0.030 \\
\hline$>=2$ a day & $24(4.2)$ & 189 (32.9) & 1 & 1 & \\
\hline \multicolumn{6}{|c|}{ Use soap for face washing } \\
\hline No & $65(11.3)$ & $265(46.5)$ & $2.48(0.241-0.68)$ & $2.3(1.32-4.12)$ & 0.004 \\
\hline Yes & $22(3.8)$ & $222(38.7)$ & 1 & 1 & \\
\hline \multicolumn{6}{|l|}{ Family size } \\
\hline $1-5$ & $19(3.3)$ & $168(29.3)$ & 1 & 1 & \\
\hline Above 5 & $68(11.8)$ & $319(55.6)$ & $0.53(1.1-2.3 .24)$ & $1.97(1.06-3.67)$ & 0.03 \\
\hline
\end{tabular}


supported that solid wastes disposed on open field was associated with presence of active trachoma [13]. This could be explained by the fact that disposing a solid waste on open field attracts more vectors of trachoma.

Families that didn't use latrine were more likely to be affected by active trachoma than those families who utilize latrine. This finding is consistent with a study conducted in Gonji Kolella district, and Gondar zuria district of Ethiopia [21, 23].

Children living in $\mathrm{HH}$ with family size greater than five were more likely to develop active trachoma than children living in $\mathrm{HH}$ less or equal to five family sizes. This finding is similar with studies conducted in Amhara region; Ethiopia $[14,15,23]$. The possible justification for this could be overcrowding living conditions among family members. This finding shows that limiting number of children is important things to prevent active trachoma.

The current study also identified that children who do not used soap for face washing were more likely to develop active trachoma than those who used soap for face washing. This is supported by previous studies conducted in Baso Liben, Laku town, Gondar zuria and Dangla, Ethiopia [9, 15-17]. Parents or caretaker should use or make child to use soap for face washing. Hygiene promotion is effective where there is good availability of water. It is even more effective when it promotes the use of soap or ash with hand and face washing [28]. Therefore, concerned bodies should do more on availing water and other sanitation facilities before promoting hygiene.

Furthermore, children who washed their face once a day had more chance to have active trachoma than children washed for two or more times in a day. This is consistent with previous studies done in Gondar zuria district [23], Gazegibella district [14] and Gonji Kolella district [21], and Wollo, Ethiopia [29]. The possible explanation for this could be the fact that frequent face washing can interrupts contact between children and trachoma vectors.

This study has some limitations which have to be taken into consideration while interpreting the findings. Methods used to measure distance from house to latrine, distance to a water source, and amount of water were not precise so that results could have been over or underestimated.

As being cross-sectional in the design, this study lacks to show temporal relationship between cause and effect.

Some variables such as cleanness of child face and fingers were taken based on observation during data collection, so that these variables could be subjected to observer's bias. Face washing frequency of children could be subjected to social desirability bias.

The sample size used in current study was low for the purpose of estimating the prevalence of $\mathrm{TF}$, as evidenced by WHO guidance, but the guidance was published after the study was done [30].

\section{Conclusion}

The present study publicized high prevalence of active trachoma among children aged 1 to 9 years. Nonutilization of latrine, absence of solid waste pits, face washing habits of children, large family size and do not use of soap for face washing were factors associated with active trachoma. The district health office and other relevant stakeholders should cooperatively work on mass distribution of antibiotic to reduce the burden of active trachoma. Implementation of SAFE strategy should be strengthened by all concerned bodies.

Study done on sanitation and water supply coverage thresholds associated with active trachoma suggests getting sufficient water and sanitation can be vital components in working towards the 2020 target of eliminating trachoma as a community health problem. Therefore, concerned bodies should do more on availing enough water and sanitation facilities [31].

\section{Additional file}

Additional file 1: English version of the questionnaires.

\section{Abbreviations}

C: Chlamydia; HH: Household; IECW: Integrated Eye Care Workers;

SAFE: Surgery, Antibiotic, Facial cleanliness and Environmental sanitation; SPSS: Statistical package for social science; TF: Trachomatous Follicular;

TI: Trachomatous Inflammation; WHO: World health organization

\section{Acknowledgements}

We would like to acknowledge Orbis International Ethiopia for tetracycline ointment and binocular lope support. We sincerely appreciate all data collectors, study participants, supervisors for their co-operation during data collection. Our thanks also go to Hawassa University and Ethiopian public health institute and the United States Center for Disease Control and Prevention for their logistic support.

\section{Authors' contribution}

EW wrote the proposal, participated in data collection, analyzed the data and drafted the paper. DD and DL approved the proposal, participated in data analysis and revised subsequent draft of the paper. $T L$ and BB participated in data analysis and revised subsequent drafts of the paper. All authors read and approved the final manuscript.

\section{Funding}

Hawassa University funded this research project. The University has no role in study design, data collection and analysis, decision to publish, or preparation of this manuscript.

\section{Availability of data and materials}

The dataset used and/or analyzed during the current study are available from the corresponding author on reasonable request.

\section{Ethics approval and consent to participate}

Ethical clearance was obtained from Ethical review committee of Hawassa University, College of medical and health science (Ref.No:IRB/065/10). Parents or caregivers were entitled to provide consent on the behalf of children 1-9 years old. Hence, verbal consent was obtained from parent or caregiver after the brief description had been given on benefits, objectives, procedure, and risk of the study. The verbal consent was obtained because written consent demanded a 
certain level of education to read and sign the consent. This was approved by ethical review committee. The reason for the eye examination and what the examination will involve was explained to parents. Confidentiality and privacy of every respondent's information was assured by not using any identifiers of the study participants. Finally, the children with active trachoma were treated with two tube of tetracycline eye ointment.

\section{Consent for publication}

"Not applicable" in this section.

\section{Competing interests}

The authors declare that they have no competing interests.

\section{Author details}

${ }^{1}$ Public health emergency management, Hadiya zone health department, Hossana, Ethiopia. ${ }^{2}$ Department of medical laboratory, Hawassa University, Hawassa, Ethiopia. ${ }^{3}$ Department of epidemiology, Hawassa University, Hawassa, Ethiopia. ${ }^{4}$ Department of nursing, Wachemo University, Durame campus, Durame, Ethiopia.

Received: 6 March 2019 Accepted: 23 September 2019

Published online: 24 October 2019

\section{References}

1. Hadfield J, Harris SR, Seth-Smith HMB, Parmar S, Andersson P, Giffard PM, et al. Comprehensive global genome dynamics of chlamydia trachomatis show ancient diversification followed by contemporary mixing and recent lineage expansion. Genome Res. 2017;27(7):1220-9.

2. Gambhir M, Basáñez MG, Burton MJ, Solomon AW, Bailey RL, Holland MJ, et al. The development of an age-structured model for trachoma transmission dynamics, pathogenesis and control. PLoS Negl Trop Dis. 2009; 3:e462.

3. WHO. Trachoma controls a guide for programme managers. Geneva: WHO; 2006

4. Center TC. Women and trachoma achieving gender equity in the implementation of SAFE; 2009.

5. Communicable disease network Australia. CDNA national guidelines for the public health management of trachoma; 2014

6. Bourne RRA, Flaxman SR, Braithwaite T, et al. Magnitude, temporal trends, and projections of the global prevalence of blindness and distance and near vision impairment: a systematic review and meta-analysis. Lancet Glob Health. 2017;5(9):e888.

7. WHO. Weekly epidemiological record. WHO. 2018;93(26):371-80

8. Oswald WE, Stewart AEP, Kramer MR, Endeshaw T, Zerihun M, et al. Active trachoma and community use of sanitation, Ethiopia. Bull World Health Organ. 2017:95:250-60.

9. Gedefaw M, Shiferaw A, Alamrew Z, Feleke A, Fentie T, Atnafu K. Current state of active trachoma among elementary school students in the context of ambitious national growth plan: the case of Ethiopia. Health. 2013;5: 1768-73.

10. Ejigu M, Kariuki MM, llako DR, Gelaw Y. Rapid Trachoma Assessment in Kersa district, Southwest Ethiopia. Ethiop J Health Sci. 2013;23(1):1-9.

11. Adera TH, Macleod C, Endriyas M, Dejene M, Willis R, BChu BK, et al. Prevalence of and risk factors for trachoma in southern nations, nationalities, and peoples' region, Ethiopia: results of 40 population-based prevalence surveys carried out with the global trachoma mapping project. Ophthalmic Epidemiol. 2016;23(sup1):84

12. Mengistu K, Shegaze M, Woldemichael K, Gesesew $H$, Markos $Y$. Prevalence and factors associated with trachoma among children aged 1-9 years in Zala district, Gamo Gofa zone, southern Ethiopia. Clin Ophthalmol. 2016;10:1663.

13. Admasu W, Hurissa BF, Benti AT. Prevalence of trachoma and associated risk factors among Yello elementary school students, in Loma Woreda, Dawro zone, Ethiopia. J Nurs Care. 2015;S1:2167.

14. Anteneh ZA, Getu WY. Prevalence of active trachoma and associated risk factors among children in Gazegibela district of Wagehemra Zone, Amhara region, Ethiopia. Trop Dis Travel Med Vaccines. 2016;2:5.

15. Shiferaw D, Moges HG. Risk factors for active trachoma among children aged 1-9 years in Maksegnit town, Gondar Zuria District, Northwest Ethiopia. Saudi J Health Sci. 2013;2(3):202.
16. Ketema K, Tiruneh M, Woldeyohannes D, Muluye D. Active trachoma and associated risk factors among children in Baso Liben District of east Gojjam, Ethiopia. BMC Public Health. 2012;12:1105.

17. Abuka T, Jamber D. Prevalence and distribution of active trachoma among children 1-9 years old at Leku town, southern Ethiopia. Curr Pediatr Res. 2017;21(3):507-13.

18. Muluneh EK, Zewotir T, Bekele Z. Rural children active trachoma risk factors and their interactions. Pan africa medical J. 2016;24:128.

19. Golovaty I, Jones L, Gelaye B, Tilahun M, Belete H, Kumie A, et al. Access to water source, latrine facilities and other risk factors of active trachoma in Ankober, Ethiopia. PLoS One. 2009;4(8):e6702.

20. Ferede AT, Dadi AF, Tariku A, Adane AA, Ayanaw T. Prevalence and determinants of active trachoma among preschool-aged children in Dembia District. Infect Dis Poverty. 2017;6:128.

21. Adane N, Berhe R, Gedefaw M. Prevalence and associated factors of active trachoma among children aged 1-9 years in rural communities of Gonji Kolella district, west Gojjam zone, North West Ethiopia. BMC Res Notes. 2015;8:641.

22. Mpyet C, Goyol M, Ogoshi C. Personal and environmental risk factors for active trachoma in children in Yobe state, North-Eastern Nigeria. Tropical Med Int Health. 2010;15(2):168-72.

23. Asres $M$, Endeshaw M, Yeshambaw M, Muluken A. Prevalence and risk factors of active trachoma among children in Gondar Zuria District North Gondar, Ethiopia. Prev Med. 2016;1(1):5

24. Yalew KN, Mekonnen MG, Jemaneh AA. Trachoma and its determinants in mojo and Lume districts of Ethiopia. Pan Afr Med J. 2012;13(1):8.

25. WHO. Report of the 18th meeting of the Who Alliance for the global elimination of Trachoma by 2020. Geneva: WHO; 2014.

26. WHO. Weekly Epidemiological Record, vol. 92. Geneva: WHO; 2017. p. 359-68.

27. Berhane Y, Worku A, Bejiga A, Adamu L, Alemayehu W, Bedri A, et al. Prevalence of Trachoma in Ethiopia. Ethiop J Health Dev. 2007;21(3):211-5.

28. Emerson P, Frost L, Bailey R, Mabey D. Implementing the safe strategy for trachoma control. A toolbox of interventions for promoting facial cleanliness and environmental improvement; 2006

29. Tadesse A, Worku A, Kumie A, Yimer SA, Beselam T. The burden of and risk factors for active trachoma in the north and south Wollo zones of Amhara region, Ethiopia. Infect Dis Poverty. 2017:6:143.

30. World Health Organization. Design parameters for population-based trachoma prevalence surveys (WHO/HTM/NTD/PCT/2018.07). Geneva: World Health Organization; 2018.

31. Garn JV, Boisson S, Willis R, Bakhtiari A, Al-Khatib T, Amer K, et al. Sanitation and water supply coverage thresholds associated with active trachoma: Modeling cross-sectional data from 13 countries. PLoS Negl Trop Dis. 2018; 12(1):e0006110.

\section{Publisher's Note}

Springer Nature remains neutral with regard to jurisdictional claims in published maps and institutional affiliations.

\section{Ready to submit your research? Choose BMC and benefit from:}

- fast, convenient online submission

- thorough peer review by experienced researchers in your field

- rapid publication on acceptance

- support for research data, including large and complex data types

- gold Open Access which fosters wider collaboration and increased citations

- maximum visibility for your research: over $100 \mathrm{M}$ website views per year

At BMC, research is always in progress.

Learn more biomedcentral.com/submission 